\title{
Evaluation of design support tools for additive manufacturing and conceptualisation of an integrated knowledge management framework
}

\author{
Claudius Ellsel ${ }^{1 *}$, Sebastian Werner ${ }^{2}$, Jan Göpfert ${ }^{3}$, Rainer Stark ${ }^{1}$ \\ ${ }^{1}$ Chair of Industrial Information Technology, Technische Universität Berlin \\ 2 Chair of Methods for Product Development and Mechatronics, Technische Universität Berlin \\ ${ }^{3}$ Institute of Energy and Climate Research, Techno-economic Systems Analysis (IEK-3), Forschungszentrum \\ Jülich
}

* Corresponding Author:

Claudius Ellsel

Technische Universität Berlin, Fakultät V - Verkehrs- und Maschinensysteme

Institut für Werkzeugmaschinen und Fabrikbetrieb, Fachgebiet Industrielle Informationstechnik

Sekretariat PTZ 4, Pascalstraße 8-9, 10587 Berlin

Phone: +491634205932

E-Mail: ellsel@tu-berlin.de

\begin{abstract}
Over time, additive manufacturing (AM) has gained more and more adoption into series production. Software tools are developed to assist designers to incorporate specific design for additive manufacturing (DfAM) guidelines into their product development processes.

In this paper, an overview of already existing DfAM functionality is given by a review of scientific publications and commercial software products.

Additionally, interviews with members of development teams which design AM parts are carried out to assess specific workflows and problems in the current design process.

In both investigations, the lack of an integrated and automated software solution emerged. Based on these results, a structure for an integrated design support toolchain is proposed.
\end{abstract}

\section{Keywords}

DfAM, review, design support tool, knowledge management, framework 


\section{Introduction}

Over time, additive manufacturing (AM) has gained more and more adoption. The generative process offers new potentials for product design. However, there are various process specific restrictions. These potentials, along with the restrictions, require special awareness and skills of a designer creating parts for AM. [1-4]

This has led to various work in the field of specific design for additive manufacturing (DfAM) guidelines [1, 3, 5-13]. These guidelines and frameworks often consist of empirical knowledge and specific advises or constraints. Consequently, their guidance for designers is of high complexity, when detailed design support is needed. To overcome this problem, software tools can be used to accompany the already existing guidelines. This allows them to be applied in a more interactive and context-sensitive way as partly described by Goguelin et al. [14].

Therefore, incorporating DfAM functionality into CAD systems is done by all major software vendors. Nevertheless, an integrated and holistic support from CAD to manufacturing does not exist.

In order to address the research questions "Which DfAM related software functionality exists?" and "What issues are present in the current toolchain?", a survey of existing software functionality is conducted, and missing functionality is identified. Additionally, engineers involved in the development of components for high temperature applications using laser powder bed fusion (LPBF) are interviewed to assess specific workflows and problems in the current design process, especially those that are related to software.

Eventually, based on the review and the interviews, a framework for an integrated design support toolchain is proposed.

\section{Review of available software support for DfAM}

\subsection{Review of publications}

Various topics connected to software support for DfAM can be identified in the literature. This review focusses on software approaches for knowledge management and DfAM analyses of parts. Other reviews already cover the optimization of the build orientation [15], the optimization of the part structure and design [16], the generation of support structures [17], shape deviation [18], or slicing and path planning [19]. Also, in [20] a general review concerning the whole DfAM process is available. However, this review is not exhaustive and does not cover knowledge management and design analyses related to DfAM.

\subsubsection{Knowledge management}

Several research activities address the management of knowledge related to $A M$ and specifically DfAM. In this context, different ontologies and knowledge bases (KBs) have been developed.

The ontology presented by Yim and Rosen connects design parameters to machine capabilities [21]. The same group introduces an ontology-based procedure for optimizing AM process plans in Liu and Rosen [22].

Eddy et al. extend an existing framework for conventional process planning with AM. The knowledge system allows to find suitable manufacturing processes for products. [23]

Dinar and Rosen develop an ontology specifically for DfAM knowledge [24]. Based on this, Kim et al. propose ontologies which link design to process parameters and model design rules $[25,26]$.

Hagedorn et al. use several ontologies to support the usage of the specific capabilities of AM in product development [27]. Qi et al. propose several linked ontologies for AM related design and processes using category theory [28]. More general ontologies considering the product life cycle around AM are presented by Mohd Ali et al. [29]. 
Sanfilippo et al. create an ontology that does not rely on other AM related ontologies due to identified problems with them [30].

To deal with uncertainty during the design process, Wang et al. introduce a knowledge management system that models probabilistic relationships with Bayesian networks [31].

Han and Schaefer propose an ontology which links machine capabilities with CAD model parameters [32]. Winkler et al. present a similar, more practical and concrete approach using SysML. Properties from AM machines are used to derive restrictive design parameters. The parameters can be transferred to a CAD system for part analysis (see 2.1.2.3) using a CSV file. [33]

In summary, there are several existing approaches covering different aspects of knowledge management in the field of DfAM. Applications are mostly presented as case studies where the knowledge is retrieved manually. There are two early approaches to link a KB with a CAD system for part analysis [32, 33]. However, the described linkage is either rather abstract or has practical limitations.

\subsubsection{DfAM design analysis}

Besides the above-described approaches for knowledge management, tools that check the alignment of parts with DfAM guidelines (design checkers) form another broad area of DfAM software support. These tools offer different stages of automation. Some require the user to input details regarding the part geometry [34, 35]. However, most software-based approaches use automated analyses to retrieve the required part parameters.

It can be distinguished between universal approaches, mesh-based analyses and CADintegrated analyses.

\subsubsection{Universal approaches}

A larger group of rather low-level approaches exist which apply geometric operations to check the manufacturability of a part.

Telea and Jalba use a voxel representation to find thin areas that are critical to manufacture [36]. Tedia and Williams use a different technique to identify critical areas in voxel representations and additionally check for critical negative structures like holes [37]. Ghiasian et al. also use voxel representations including methods from [36] and integrate the analysis into a larger framework [38].

Design checks based on individual layers are introduced by Nelaturi et al. [39] as well as Jaiswal and Rai [40]. Both additionally offer methods to automatically correct critical areas [39, 40]. Since only layers are analysed, critical areas that do not occur in plane but in other directions likely cannot be identified.

\subsubsection{Mesh-based analysis}

More practical approaches are using polygon-meshes as input format for the analysis.

Lu introduces a method that extracts parameters from polygon-meshes and compares them with machine properties. As an example, the bounding box is mentioned. Additionally, machine learning is used to identify manufacturable parts. [41] However, it is not clear, whether the bounding box is the only feature that is checked.

Shi et al. employ the heat kernel signature of a part to identify features that are critical to manufacture [42].

Chen and Xu use an offsetting algorithm on polygonal models to identify critical features in parts [43]. Other approaches checking further criteria such as part size or overhang angles are presented in [44], [45] and as part of a larger framework including optimization of the build orientation in [46]. Tominski et al. connect a KB with the analysis to provide information about 
critical parameters [47]. Building upon the approach of [44], Mayerhofer et al. likewise propose a framework that connects the analysis with a KB. Furthermore, the solution is integrated into a cloud platform. [48] In a consecutive publication, the results of the implemented framework are presented [49].

\subsubsection{CAD-integrated analysis}

The following approaches check the design of CAD parts within a CAD environment.

Ranjan et al. introduce a feature graph based method to assess manufacturability in which individual part features are identified and analysed within Siemens NX. To support this, a producibility index is used. However, only some analysis steps are directly performed on the CAD model. For the rest, other techniques are used like analysing slices from a derived mesh model. [50] Zhang et al. present layer based DfAM analyses as part of several AM related tools for Siemens NX. Further provided functionality includes the generation of support structures and optimization of the build orientation. They also use a producibility index as introduced in [50]. [51] Campana and Mele provide another approach that analyses features. It is integrated into the CAD environment CimatronE by 3D Systems. The toolset also includes a GUI to select different machines from an integrated database affecting the critical design parameters for the analysis. [52] Winkler et al. introduce an automated part analysis in Siemens NX. An index to assess manufacturability is proposed. Additionally, identified critical features can be visualised. [53]

Tendencies to make use of a KB as observed for mesh-based analyses can be seen here as well, but to a much lesser extent. Layer or slice-based analysis methods probably have the same problems as described for the universal approaches (see 2.1.2.1).

The review showed many approaches in scientific publications related to DfAM support tools in the domains of knowledge management and evaluation of specific design parameters. However, these approaches oftentimes mainly describe general methods and are rather isolated. There are only few tools that are integrated in CAD software. In those cases, there is no universal integration with a KB.

\subsection{Review of commercially available software functionality}

DfAM functionality is provided by several commercial software products, either directly integrated in CAD systems or as specialized standalone software. The latter can often be integrated into CAD software via plugins. Software products that can be used in different stages of the DfAM process have been listed before [20]. However, only few of the available tools are mentioned and the functionality is not analysed.

For the analysis, software tools for which information was accessible and that have a broad DfAM functional scope were selected. Table 1 shows a comparison regarding functionality of the respective tools. The information is based on official information related to the latest and most advanced versions of the tools. Additionally, available software was installed, and further investigation was conducted (Siemens NX 1980, PTC Creo 7, Autodesk Netfabb 2021, Additiveworks Amphyon 2021, Simufact Additive 2021).

The functionality has been categorized into the following relevant sections that are mostly aligned in order with the actual design process: CAD-integrated describes, whether the software is a CAD software, can be integrated into CAD software via a plugin or can only be used standalone. The first relevant process step is the file import. Here the variety of supported import formats and possible repair options are considered. The next relevant functionality is the ability to check the part design for compliance with DfAM guidelines. Depending on the tool, this can be helpful to identify potential problems already early in the design process. During the design phase other functionality like topology optimization (possibly with AM 
specific considerations) can be used. Either still during the design phase within the CAD system or during the manufacturing preparation steps, lattice structures can be generated. For the manufacturing preparation, the tools often offer automatic part placement and nesting options. Another function for manufacturing preparation is to optimize the part orientation. Then, the generation of support structures can be executed and lastly often tools offer a build process simulation to identify problems and compensate distortions.

Table 1: Comparison of commercial software functionality

\begin{tabular}{|c|c|c|c|c|c|c|c|c|c|}
\hline Software & 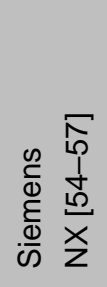 & 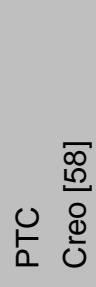 & 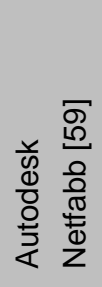 & 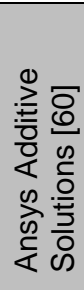 & 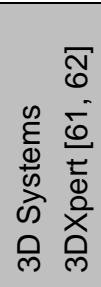 & 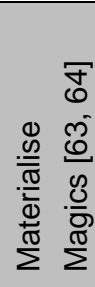 & 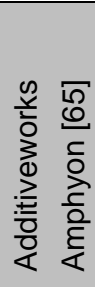 & 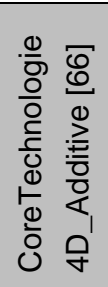 & 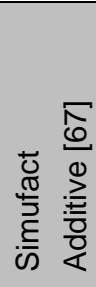 \\
\hline CAD-integrated & - & - & D & D & D & D & D & - & - \\
\hline File import & ○ & - & - & ○ & • & • & D & ○ & - \\
\hline Design check & D & D & D & - & D & D & - & D & - \\
\hline $\begin{array}{l}\text { Topology } \\
\text { optimization }\end{array}$ & (1) & (1) & (1) & $\bullet$ & - & - & - & - & - \\
\hline $\begin{array}{l}\text { Lattice structure } \\
\text { generation }\end{array}$ & - & ○ & ○ & 0 & $\bullet$ & 0 & - & 0 & - \\
\hline $\begin{array}{l}\text { Part placement / } \\
\text { nesting }\end{array}$ & $\bullet$ & $\bullet$ & $\bullet$ & 0 & $\bullet$ & $\bullet$ & - & $\bullet$ & (1) \\
\hline Part orientation & ○ & D & ○ & ○ & $\bullet$ & - & $\bullet$ & 0 & • \\
\hline $\begin{array}{l}\text { Support structure } \\
\text { generation }\end{array}$ & - & D & ○ & 0 & • & 0 & ○ & • & 0 \\
\hline $\begin{array}{l}\text { Process simulation / } \\
\text { compensation }\end{array}$ & $\bullet$ & (1) & $\bullet$ & 0 & 0 & 0 & 0 & - & 0 \\
\hline
\end{tabular}

For many categories, functionality is broadly available in the compared products. This is not the case for the design check and topology optimization. For the latter this might be explained by its more complex functionality which is also not limited to AM. In CAD software like Siemens $N X$ and PTC Creo it is included as part of the other functionalities of those software suites. For design checks, often only limited functionality was found not covering all relevant geometric features.

In addition to the listed functionality some software offers machine and or material databases. This can for example change the available build volume or be used for process simulation. However, these databases do not connect to design check threshold values and are not integrated into more sophisticated concepts like digital master models.

\subsection{Interviews}

To identify interruptions, time-consuming loops and missing software functionality in current development processes for LPBF in high temperature applications, interviews with engineers from AM application, heat exchange design, business administration and development engineers were conducted. In total, eleven people participated. The interview focused on repetitive tasks, identification of waiting times due to lack of information and missing information communication. 
The interviews gave several insights from the user perspective. Complex design process workflows and interactions are found to be very time consuming. Also, demand is raised to automate repetitive, sometimes rather tedious tasks like support structure creation and checking for sharp edges especially when switching between different software for CAD and buildjob preparation. AM enables functional integration which leads to significantly increased size and complexity of CAD models compared to parts designed for conventional manufacturing. This in turn results in increased review and handling efforts. A further effect is the demand for cost estimation early in the design process, to justify designing for AM. Further typical problems are different modelling strategies between company departments and communication of requirements between design and AM application engineers in cases where feature geometry is driven by functionality rather than printability. Material and machine dependent restrictions and possibilities, apart from general design guidelines, are not available to every stakeholder in the design process. Additionally, a certain level of standardization of design features like for conventional machining is not achieved yet. Lastly, it was observed, that occasionally knowledge about existing software functionality is lacking.

In conclusion, there is a great potential for an integrated and automated software solution to enhance product development. Switching between different tools in iterative loops could be omitted and information availability throughout the company would improve.

\section{Proposal of an integrated approach}

The review showed a lack of CAD-integrated DfAM solutions that are linked to a central knowledge base (KB). On the other hand, the interviews show demand to reduce complexity in the toolchain and to increase the availability of knowledge. Therefore, we propose an integrated approach which connects a KB to a design support tool within a CAD environment. This can be seen as an application or evolvement of the framework proposed by Molcho et al. [68] for the AM domain. An integrated approach like this also enables a simulation-driven design process in AM, as was demonstrated in other fields already [69, 70]. Figure 1 shows the proposed structure for this integration.

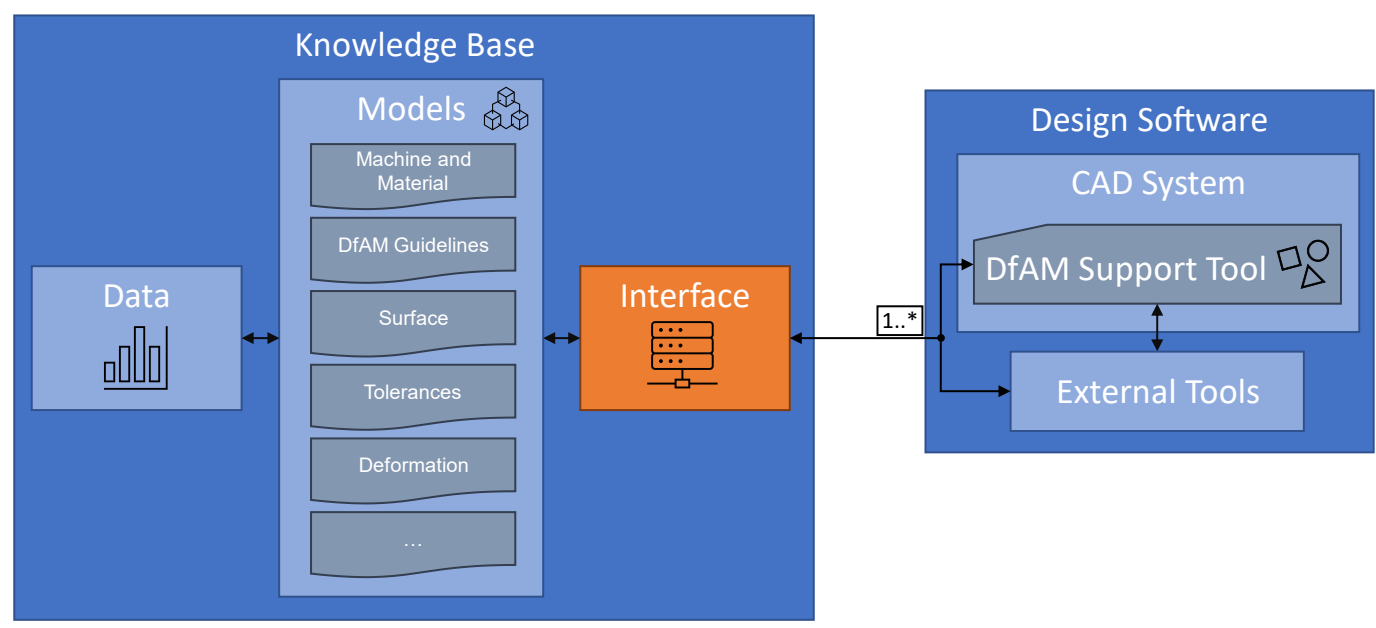

Figure 1: Schematic of the proposed integration structure

The KB contains data, rules, and models for aspects like machine and process, material, as well as DfAM guidelines. The models are linked with data and provide internal logic rules (like interpolation) to deliver required information to the design software. The data can be stored within the KB but also be retrieved from already available sources like PLM systems or other repositories for digital master and associated simulation models. The exchange of this information is organized through a central interface that provides access for the design 
software. Within the CAD system, a support tool (plugin) which is connected to said interface provides the functionalities for DfAM in combination with external tools. The vendor of the CAD system could as well provide a direct connection to the interface.

The solution combines KBs with a CAD based tool like already seen for mesh-based analysis approaches (see 2.1.2.2). The generalized interface can be realized using a REST API in practice. It allows the KB to function independently of the design software. This means several clients with design software can easily access the unified knowledge in the central KB. Restrictive design parameters, e.g., can be made available for DfAM studies through such a tool linkage. Additionally, the KB can provide further functionality like specific information on DfAM guidelines that can be offered to the user through the CAD interface for example when visualizing identified critical areas after a design check.

In comparison to existing solutions, an overall framework to link a KB to design tools is described that can easily be applied in practice and offers high flexibility through the unified interface.

\section{Conclusion and outlook}

In this paper, a review of functionality in publications and commercial software tools regarding support for DfAM has been conducted.

Both, research presented in publications and commercial software tools often lack fully integration into CAD systems. This results in disruptions in the development process. Furthermore, the usage and integration of knowledge management systems has to be extended.

Additionally, a series of interviews with different stakeholders in the development of AM components was conducted. Availability and communication of information regarding DfAM and functional features are the key factors to enhance the product development process.

In consequence, a new framework was presented to link a knowledge base for AM with CADintegrated software tools using a universal interface. This enables the communication of data from the field as well as from digital twins to stakeholders involved in the design process and serves as a basis for new functionality to automatize repetitive tasks during design evaluation.

Future work will comprise the implementation of the framework, which is currently at a conceptual stage, including the support tool to provide the DfAM functionality in the CAD system.

Authors contributions: CE surveyed the available software support; SW and JG prepared and conducted the interviews; CE wrote the initial draft; CE, SW and JG finalised the script; RS supervised the research, commented and revised the script. All authors have read and agreed to the published version of the manuscript.

Acknowledgment: Our project "WvSC - HTA 2.0: HochTemperaturAnwendungen 2.0" is funded by the European Regional Development Fund (Grant number 10167551). This work was carried out within the framework of the Werner-von-Siemens Centre for Industry and Science (https://wvsc.berlin/).

Conflicts of Interest: The authors declare no conflict of interest.

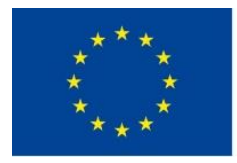

EUROPEAN UNION

European Regional Development Fund

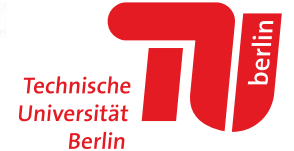

Berlin

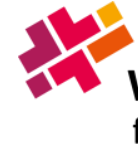

Werner-von-Siemens Centre

for Industry and Science 


\section{References}

[1] I. Gibson, D. Rosen, B. Stucker, and M. Khorasani, Additive Manufacturing Technologies. Cham: Springer International Publishing, 2021.

[2] S. Kumar, Additive Manufacturing Processes. Cham: Springer International Publishing, 2020.

[3] M. K. Thompson et al., "Design for Additive Manufacturing: Trends, opportunities, considerations, and constraints," CIRP Annals, vol. 65, no. 2, pp. 737-760, 2016, doi: 10.1016/j.cirp.2016.05.004.

[4] M. Schmidt et al., "Laser based additive manufacturing in industry and academia," CIRP Annals, vol. 66, no. 2, pp. 561-583, 2017, doi: 10.1016/j.cirp.2017.05.011.

[5] G. A. O. Adam and D. Zimmer, "On design for additive manufacturing: evaluating geometrical limitations," Rapid Prototyping Journal, vol. 21, no. 6, pp. 662-670, 2015, doi: 10.1108/RPJ-06-2013-0060.

[6] J. W. Booth, J. Alperovich, P. Chawla, J. Ma, T. N. Reid, and K. Ramani, "The Design for Additive Manufacturing Worksheet," Journal of Mechanical Design, vol. 139, no. 10, 2017, doi: 10.1115/1.4037251.

[7] O. Diegel, A. Nordin, and D. Motte, A Practical Guide to Design for Additive Manufacturing. Singapore: Springer Singapore, 2019.

[8] D. Thomas, "The Development of Design Rules for Selective Laser Melting," Ph.D. Thesis, University of Wales, Cardiff, 2009. [Online]. Available: https://repository.cardiffmet.ac.uk/handle/10369/913

[9] J. Kranz, D. Herzog, and C. Emmelmann, "Design guidelines for laser additive manufacturing of lightweight structures in TiAl6V4," Journal of Laser Applications, vol. 27, S1, S14001, 2015, doi: 10.2351/1.4885235.

[10] G. A. Adam and D. Zimmer, "Design for Additive Manufacturing-Element transitions and aggregated structures," CIRP Journal of Manufacturing Science and Technology, vol. 7, no. 1, pp. 20-28, 2014, doi: 10.1016/j.cirpj.2013.10.001.

[11] G. A. Teitelbaum, L. C. Schmidt, and Y. Goaer, "Examining Potential Design Guidelines for Use in Fused Deposition Modeling to Reduce Build Time and Material Volume," in 14th Design for Manufacturing and the Life Cycle Conference - 6th Symposium on International Design and Design Education, San Diego, California, USA, 2010, pp. 73-82.

[12] B. Vayre, F. Vignat, and F. Villeneuve, "Identification on Some Design Key Parameters for Additive Manufacturing: Application on Electron Beam Melting," Procedia CIRP, vol. 7, pp. 264-269, 2013, doi: 10.1016/j.procir.2013.05.045.

[13] T. Vaneker, A. Bernard, G. Moroni, I. Gibson, and Y. Zhang, "Design for additive manufacturing: Framework and methodology," CIRP Annals, vol. 69, no. 2, pp. 578-599, 2020, doi: 10.1016/j.cirp.2020.05.006.

[14] S. Goguelin, J. M. Flynn, and V. Dhokia, "A Bottom-up Design Framework for CAD Tools to Support Design for Additive Manufacturing," in Sustainable Design and Manufacturing 2016, 2016, pp. 411-421.

[15] L. Di Angelo, P. Di Stefano, and E. Guardiani, "Search for the Optimal Build Direction in Additive Manufacturing Technologies: A Review," JMMP, vol. 4, no. 3, p. 71, 2020, doi: 10.3390/jmmp4030071.

[16] J. Plocher and A. Panesar, "Review on design and structural optimisation in additive manufacturing: Towards next-generation lightweight structures," Materials \& Design, vol. 183, p. 108164, 2019, doi: 10.1016/j.matdes.2019.108164.

[17] J. Jiang, X. Xu, and J. Stringer, "Support Structures for Additive Manufacturing: A Review," JMMP, vol. 2, no. 4, p. 64, 2018, doi: 10.3390/jmmp2040064.

[18] Z. ZHU, S. KEIMASI, N. ANWER, L. MATHIEU, and L. QIAO, "Review of Shape Deviation Modeling for Additive Manufacturing," in Lecture Notes in Mechanical Engineering, Advances on Mechanics, Design Engineering and Manufacturing: Proceedings of the International Joint Conference on Mechanics, Design Engineering \& Advanced Manufacturing (JCM 2016), 14-16 September, 2016, Catania, Italy, B. Eynard, V. Nigrelli, S. M. Oliveri, G. Peris-Fajarnes, and S. Rizzuti, Eds., Cham: Springer International Publishing, 2016, pp. 241-250.

[19] D. Zhao and W. Guo, "Shape and Performance Controlled Advanced Design for Additive Manufacturing: A Review of Slicing and Path Planning," Journal of Manufacturing Science and Engineering, vol. 142, no. 1, 2020, doi: $10.1115 / 1.4045055$.

[20] A. Wiberg, J. Persson, and J. Ölvander, "Design for additive manufacturing - a review of available design methods and software," RPJ, vol. 25, no. 6, pp. 1080-1094, 2019, doi: 10.1108/RPJ-10-2018-0262.

[21] S. Yim and D. Rosen, "Case-Based Retrieval Approach of Supporting Process Planning in Layer-Based Additive Manufacturing," in Proceedings of the ASME International Design Engineering Technical Conferences and Computers and Information in Engineering Conference - 2007: Presented at 2007 ASME International Design Engineering Technical Conferences and Computers and Information in Engineering Conference, September 4 - 7, 2007, Las Vegas, Nevada, USA, Las Vegas, Nevada, USA, 2008, pp. 841852.

[22] X. Liu and D. W. Rosen, "Ontology Based Knowledge Modeling and Reuse Approach of Supporting Process Planning in Layer-Based Additive Manufacturing," in 2010 International Conference on Manufacturing Automation: ICMA 2010, 13-15 December 2010, Hong Kong, China, proceedings, Hong Kong, China, 2010, pp. 261-266.

[23] D. Eddy, S. Krishnamurty, I. Grosse, M. Perham, J. Wileden, and F. Ameri, "Knowledge Management With an Intelligent Tool for Additive Manufacturing," in Proceedings of the ASME International Design Engineering Technical Conferences and Computers and Information in Engineering Conference - 2015 : 
Presented at ASME 2015 International Design Engineering Technical Conferences and Computers and Information in Engineering Conference : August 2-5, 2015, Boston, Massachusetts, USA, Boston, Massachusetts, USA, 2016.

[24] M. Dinar and D. W. Rosen, "A Design for Additive Manufacturing Ontology," Journal of Computing and Information Science in Engineering, vol. 17, no. 2, 2017, doi: 10.1115/1.4035787.

[25] S. Kim, D. W. Rosen, P. Witherell, and H. Ko, "Linking Part Design To Process Planning By Design For Additive Manufacturing Ontology," in Proceedings of the 3rd International Conference on Progress in Additive Manufacturing (Pro-AM 2018), 2018, pp. 303-308.

[26] S. Kim, D. W. Rosen, P. Witherell, and H. Ko, "A Design for Additive Manufacturing Ontology to Support Manufacturability Analysis," Journal of Computing and Information Science in Engineering, vol. 19, no. 4, 2019, doi: 10.1115/1.4043531.

[27] T. J. Hagedorn, S. Krishnamurty, and I. R. Grosse, "A Knowledge-Based Method for Innovative Design for Additive Manufacturing Supported by Modular Ontologies," Journal of Computing and Information Science in Engineering, vol. 18, no. 2, 2018, doi: 10.1115/1.4039455.

[28] Q. Qi, L. Pagani, P. J. Scott, and X. Jiang, "A categorical framework for formalising knowledge in additive manufacturing," Procedia CIRP, vol. 75, pp. 87-91, 2018, doi: 10.1016/j.procir.2018.04.076.

[29] M. Mohd Ali, R. Rai, J. N. Otte, and B. Smith, "A product life cycle ontology for additive manufacturing," Computers in Industry, vol. 105, pp. 191-203, 2019, doi: 10.1016/j.compind.2018.12.007.

[30] E. M. Sanfilippo, F. Belkadi, and A. Bernard, "Ontology-based knowledge representation for additive manufacturing," Computers in Industry, vol. 109, pp. 182-194, 2019, doi: 10.1016/j.compind.2019.03.006.

[31] Y. Wang, R. Blache, P. Zheng, and X. Xu, "A Knowledge Management System to Support Design for Additive Manufacturing Using Bayesian Networks," Journal of Mechanical Design, vol. 140, no. 5, 2018, doi: 10.1115/1.4039201.

[32] J. Han and D. Schaefer, "An Ontology for Supporting Digital Manufacturability Analysis," Procedia CIRP, vol. 81, pp. 850-855, 2019, doi: 10.1016/j.procir.2019.03.211.

[33] M. Winkler, G. Jacobs, K. Spütz, and C. Konrad, "Evaluation of Parts for Additive Manufacturing utilizing System Models of AM Plants," IOP Conf. Ser.: Mater. Sci. Eng., vol. 1097, no. 1, p. 12022, 2021, doi: 10.1088/1757-899X/1097/1/012022.

[34] M. T. Samperi, "Development of design guidelines for metal additive manufacturing and process selection (2014)," Master thesis, Department of Industrial and Manufacturing Engineering, Pennsylvania State University, 2014. [Online]. Available: https://etda.libraries.psu.edu/catalog/21832

[35] R. Bibb, Z. Taha, R. Brown, and D. Wright, "Development of a rapid prototyping design advice system," $J$ Intell Manuf, vol. 10, 3/4, pp. 331-339, 1999, doi: 10.1023/A:1008920512663.

[36] A. Telea and A. Jalba, "Voxel-Based Assessment of Printability of 3D Shapes," in Lecture Notes in Computer Science, Mathematical Morphology and Its Applications to Image and Signal Processing, P. Soille, M. Pesaresi, and G. K. Ouzounis, Eds., Berlin, Heidelberg: Springer Berlin Heidelberg, 2011, pp. 393-404.

[37] S. Tedia and C. B. Williams, "Manufacturability Analysis Tool for Additive Manufacturing Using Voxel-Based Geometric Modeling," Solid Freeform Fabrication 2016: Proceedings of the 27th Annual International Solid Freeform Fabrication Symposium - An Additive Manufacturing Conference, 2016.

[38] S. E. Ghiasian, P. Jaiswal, R. Rai, and K. Lewis, "From Conventional to Additive Manufacturing: Determining Component Fabrication Feasibility," in Proceedings of the ASME International Design Engineering Technical Conferences and Computers and Information in Engineering Conference - 2018: Presented at ASME 2018 International Design Engineering Technical Conferences and Computers and Information in Engineering Conference, August 26-29, 2018, Quebec City, Canada, Quebec City, Quebec, Canada, 2018.

[39] S. Nelaturi, W. Kim, and T. Kurtoglu, "Manufacturability Feedback and Model Correction for Additive Manufacturing," Journal of Manufacturing Science and Engineering, vol. 137, no. 2, 2015, doi: 10.1115/1.4029374.

[40] P. Jaiswal and R. Rai, "A geometric reasoning approach for additive manufacturing print quality assessment and automated model correction," Computer-Aided Design, vol. 109, pp. 1-11, 2019, doi: 10.1016/j.cad.2018.12.001.

[41] T. Lu, "Towards a fully automated 3D printability checker," in 2016 IEEE International Conference on Industrial Technology (ICIT): Proceedings : the Howard Plaza Hotel Taipei, Taipei, Taiwan, 14-17 March 2016, Piscataway, NJ: IEEE, 2016, pp. 922-927.

[42] Y. Shi, Y. Zhang, S. Baek, W. de Backer, and R. Harik, "Manufacturability analysis for additive manufacturing using a novel feature recognition technique," Computer-Aided Design and Applications, vol. 15, no. 6, pp. 941-952, 2018, doi: 10.1080/16864360.2018.1462574.

[43] Y. Chen and X. Xu, "Manufactruability Analysis of Infeasible Features in Polygonal Models for Web-Based Rapid Prototyping," in 2010 International Conference on Manufacturing Automation: ICMA 2010, 13-15 December 2010, Hong Kong, China, proceedings, Hong Kong, China, 2010, pp. 120-127.

[44] J.-P. Rudolph and C. Emmelmann, "Analysis of Design Guidelines for Automated Order Acceptance in Additive Manufacturing," Procedia CIRP, vol. 60, pp. 187-192, 2017, doi: 10.1016/j.procir.2017.01.027. 
[45] S. Goguelin, J. Colaco, V. Dhokia, and D. Schaefer, "Smart Manufacturability Analysis for Digital Product Development," Procedia CIRP, vol. 60, pp. 56-61, 2017, doi: 10.1016/j.procir.2017.02.026.

[46] S. Chowdhury, K. Mhapsekar, and S. Anand, "Part Build Orientation Optimization and Neural NetworkBased Geometry Compensation for Additive Manufacturing Process," Journal of Manufacturing Science and Engineering, vol. 140, no. 3, 2018, doi: 10.1115/1.4038293.

[47] J. Tominski, S. Lammers, C. Wulf, and D. Zimmer, "Method for a Software-Based Design Check of Additively Manufactured Components," Solid Freeform Fabrication 2018: Proceedings of the 29th Annual International Solid Freeform Fabrication Symposium, 2018.

[48] M. Mayerhofer, M. Merdan, M. Schwentenwein, and W. Lepuschitz, "Manufacturability Analysis for Additive Manufacturing," 2019 24th IEEE International Conference on Emerging Technologies and Factory Automation (ETFA), pp. 1252-1255, 2019, doi: 10.1109/ETFA.2019.8868965.

[49] M. Mayerhofer, W. Lepuschitz, T. Hoebert, M. Merdan, M. Schwentenwein, and T. I. Strasser, "KnowledgeDriven Manufacturability Analysis for Additive Manufacturing," IEEE Open J. Ind. Electron. Soc., vol. 2, pp. 207-223, 2021, doi: 10.1109/OJIES.2021.3061610.

[50] R. Ranjan, R. Samant, and S. Anand, "Design for Manufacturability in Additive Manufacturing Using a Graph Based Approach," in Proceedings of the ASME 10th International Manufacturing Science and Engineering Conference - 2015: Presented at ASME 2015 10th International Manufacturing Science and Engineering Conference, June 8 - 12, 2015, Charlotte, North Carolina, USA, Charlotte, North Carolina, USA, 2015.

[51] B. Zhang, A. Goel, O. Ghalsasi, and S. Anand, "CAD-based design and pre-processing tools for additive manufacturing," Journal of Manufacturing Systems, vol. 52, pp. 227-241, 2019, doi: 10.1016/j.jmsy.2019.03.005.

[52] G. Campana and M. Mele, "An application to Stereolithography of a feature recognition algorithm for manufacturability evaluation," J Intell Manuf, vol. 31, no. 1, pp. 199-214, 2020, doi: 10.1007/s10845-0181441-8.

[53] M. Winkler, S. Stürmer, and C. Konrad, "Evaluation of the Additive Manufacturability of CAD-Parts for initial Data Labelling in Al-based Part Identification," IOP Conf. Ser.: Mater. Sci. Eng., vol. 1097, no. 1, p. 12020, 2021, doi: 10.1088/1757-899X/1097/1/012020.

[54] Siemens, Design for Additive Manufacture. [Online]. Available: https://www.plm.automation.siemens.com/ global/en/products/mechanical-design/design-for-additive-manufacture.html (accessed: Jun. 24 2021).

[55] Siemens, Build Preparation. [Online]. Available: https://www.plm.automation.siemens.com/global/en/ products/manufacturing-planning/build-preparation.html (accessed: Jun. 24 2021).

[56] Siemens, AM Generative Engineering. [Online]. Available: https://www.plm.automation.siemens.com/global/ en/products/manufacturing-planning/generative-engineering.html (accessed: Jun. 24 2021).

[57] Siemens, Build Simulation. [Online]. Available: https://www.plm.automation.siemens.com/global/en/ products/manufacturing-planning/build-simulation.html (accessed: Jun. 24 2021).

[58] PTC, "Additive manufacturing capabilities in Creo (2021)," 2021. Accessed: Jun. 24 2021. [Online]. Available: https://www.ptc.com/-/media/Files/PDFs/CAD/Creo8/additive-manufacturing-brochure/additivemanufacturing-capabilities-in-creo-brochure-en.PDF

[59] Autodesk Inc., Compare products - Fusion 360 with Netfabb additive manufacturing solutions. [Online]. Available: https://www.autodesk.com/compare/compare-features/netfabb (accessed: Jun. 24 2021).

[60] ANSYS, Inc., Ansys Additive Manufacturing. [Online]. Available: https://www.ansys.com/products/additive (accessed: Jun. 24 2021).

[61] 3D Systems, Inc., Features. [Online]. Available: https://www.3dsystems.com/software/3dxpert/features (accessed: Jun. 24 2021).

[62] Solid Solutions Technical Team, 3DXpert for SOLIDWORKS. [Online]. Available: https:// blogs.solidworks.com/tech/2018/05/3dxpert-for-solidworks.html (accessed: Jul. 3 2021).

[63] Materialise, Product information. [Online]. Available: https://www.materialise.com/software/magics/productinformation (accessed: Jun. 24 2021).

[64] Materialise, Magics. [Online]. Available: https://www.materialise.com/software/magics (accessed: Jul. 2 2021).

[65] Additive Works GmbH, Overview. [Online]. Available: https://additive.works/overview (accessed: Jun. 24 2021).

[66] CT CoreTechnologie Group, "4D Additive Brochure," 2020. Accessed: Jun. 25 2021. [Online]. Available: https://www.coretechnologie.com/fileadmin/content/Broschueren/4D_Additive_en.pdf

[67] simufact engineering gmbh, Functions in Simufact Additive. [Online]. Available: https://www.simufact.com/ funktional-description.html (accessed: Jun. 24 2021).

[68] G. Molcho, Y. Zipori, R. Schneor, O. Rosen, D. Goldstein, and M. Shpitalni, "Computer aided manufacturability analysis: Closing the knowledge gap between the designer and the manufacturer," CIRP Annals, vol. 57, no. 1, pp. 153-158, 2008, doi: 10.1016/j.cirp.2008.03.046.

[69] S. Harries and C. Abt, "CAESES-The HOLISHIP Platform for Process Integration and Design Optimization," in A Holistic Approach to Ship Design, A. Papanikolaou, Ed., Cham: Springer International Publishing, 2019, pp. 247-293.

[70] A. Papanikolaou et al., "A Holistic Approach to Ship Design: Tools and Applications," Journal of Ship Research, pp. 1-29, 2020, doi: 10.5957/JOSR.12190070. 